\title{
Identifying Regional Cardiac Abnormalities from Myocardial Strains Using Spatio-temporal Tensor Analysis
}

\author{
Zhen Qian ${ }^{1}$, Qingshan Liu ${ }^{1}$, Dimitris N. Metaxas ${ }^{1}$, and Leon Axel ${ }^{2}$ \\ ${ }^{1}$ Center for Computational Biomedicine Imaging and Modeling (CBIM), Rutgers \\ University, New Brunswick, NJ, USA \\ ${ }^{2}$ Department of Radiology, New York University, New York, NY, USA
}

\begin{abstract}
Myocardial deformation is a critical indicator of many cardiac diseases and dysfunctions. The goal of this paper is to use myocardial deformation patterns to identify and localize regional abnormal cardiac function in human subjects. We have developed a novel tensor-based classification framework that better conserves the spatio-temporal structure of the myocardial deformation pattern than conventional vector-based algorithms. In addition, the tensor-based projection function keeps more of the information of the original feature space, so that abnormal tensors in the subspace can be back-projected to reveal the regional cardiac abnormality in a more physically meaningful way. We have tested our novel method on 41 human image sequences, and achieved a classification rate of $87.80 \%$. The recovered regional abnormalities from our algorithm agree well with the patient's pathology and doctor's diagnosis and provide a promising avenue for regional cardiac function analysis.
\end{abstract}

\section{Introduction}

Myocardial deformation (or strain) is an important early indicator of many cardiac diseases and dysfunctions, such as ischemia and infarction. Recently, the technique of MRI tagging (tMRI) [1] has been extensively used in research and clinical applications to extract detailed information on myocardial deformation in vivo. This technique generates sets of equally spaced tagging lines or grids within the myocardium as temporary material markers, through spatial modulation of the magnetization. For deformation extraction from the images, many motion and strain estimation methods have been proposed for tMRI. In [2, deformed tags were tracked and interpolated using a spline method to obtain a dense displacement map, from which the 2D Lagrangian strain was calculated. In 3. the spatio-temporal continuity constraint was used to derive the myocardial displacement field. In [4], the myocardial velocity field and pathlines were calculated from the phase map using the HARP technique. Then the strain was obtained from the HARP phase tracking results. The left ventricle was divided into 8 sectors and regional myocardial strains were calculated, which demonstrated the inhomogeneous strain distribution in the ventricle. In [567], $3 D+t$

D. Metaxas et al. (Eds.): MICCAI 2008, Part I, LNCS 5241, pp. 789-797, 2008.

(C) Springer-Verlag Berlin Heidelberg 2008 
strains were derived from 3D parametrical deformable models using either FEM or B-Spline tensor techniques.

Some of the aforementioned strain estimation methods 26 6] have been implemented to compare normal and pathological data. Their results show noticeable differences in the strain pattern of normal and abnormal hearts, which reveals the potential of using strain to identify pathology in cardiac function. However, limited research has been conducted to fulfill this potential in a quantitative manner. In [8], an exploratory normal contraction reference model has been set up, based on the principal component analysis (PCA) of tMRI scans from 8 healthy subjects using their spatio-temporal deformation. Abnormalities could be found by comparing with the reference model in the PCA subspace.

The goal of this paper is to use the spatio-temporally distributed myocardial strain pattern to identify and localize abnormal cardiac function in human subjects. The main difficulty behind this is the large strain variance among normal subjects, and the even larger variance in patients, which makes it difficult to define a normal or abnormal criterion. On the other hand, the spatio-temporally distributed myocardial strain is far more complicated to quantitatively interpret than the 1D ECG signal or the scalar ejection fraction. Pathology develops with complicated and systematic consequences. For example, a region of ischemia might cause the rest of the heart to contract more vigorously to compensate. In this case, the spatial or temporal pattern of the strain could be of more importance than strain value alone in detecting cardiac malfunctions.

In this paper, to solve these two difficulties, we formulate the detection of abnormality into a novel spatio-temporal tensor-based linear discriminant analysis (LDA) classification framework. In order to learn the classifier in a supervised fashion, we gathered a group of normal and patient tMRI sequences acquired in short axis (SA), and applied a non-tracking-based strain estimation method [9] to extract the radial strain, circumferential strain and tissue rotation angles to construct the training data. Then a tensor-based LDA classifier was employed, based on the Fisher criterion to find an optimal linear projection that maximizes the between-class, i.e., the normal and patient, scatter and minimizes the within-class scatter. Compared with the vector-based conventional LDA that projects the feature space onto a scalar, the advantage of using our spatio-temporal tensor-based LDA is that its dimension reduction is separately operated in the spatial and temporal domains, so that it conserves better the spatio-temporal structure and information of the training data. On the other hand, the advantage of using a linear classifier, rather than nonlinear approaches such as SVM, is that by using the pseudo-inverse matrix of the linear projection function, we are able to back-project the abnormality found in the subspace to the original feature space, so as to localize the regional cardiac abnormality in a more physically meaningful way. We have tested our novel classification algorithm on a clinical dataset of forty one tMRI sequences from normal and patient subjects, and have achieved a higher or comparable detection accuracy rate compared to conventional classification algorithms, such as PCA, LDA and SVM. The recovered regional abnormalities from our algorithm agree well with 
the patient's pathology and doctor's diagnosis and provide a promising avenue to regional cardiac function analysis.

\section{Methodology}

\subsection{Strain Estimation and Data Preparation from tMRI}

Non-tracking-based strain estimation. We adapt a non-tracking-based strain estimation method [9] to extract the motion information from the tMRI sequences. We assume that the myocardium is incompressible, and that it undergoes three possible deformations: stretching, compression, and local rotation. Instead of calculating the strain values in terms of the gradient of the displacement by tracking the tag pattern [2] or the embedded tag phase 4, this nontracking based method directly analyzes the tag deformation gradient by locally applying a 2D Gabor filter and optimizing its parameters, i.e., the orientation $\phi$ and spacing $S$ of a 2D Gabor's sinusoidal modulation in the spatial domain. This optimization procedure can be applied in each temporal frame independently, so that it can avoid the tracking step, which might be problematic when temporal resolution is relatively low and "tag-jump" can occur.

See Fig. 1 (a) for an illustration. The initial tag spacing $D_{x}=D_{y}=D$ and orientation $\phi_{o}$ are referred to as the initial state, so that the Lagrangian deformation gradient in the beating myocardium can be obtained by comparing the deformed tag spacing $S$ and orientation change $\Delta \phi$ to the initial state. A $2 \mathrm{D}$ deformation gradient tensor $\mathbf{F}$ can be derived by:

$$
\mathbf{F}=\left[\begin{array}{ll}
\frac{S_{x} \cos \Delta \phi_{y}}{D \sin \phi} & \frac{S_{y} \sin \Delta \phi_{x}}{D \sin \phi} \\
\frac{S_{x} \sin \Delta \phi_{y}}{D \sin \phi} & \frac{S_{y} \cos \Delta \phi_{x}}{D \sin \phi}
\end{array}\right]
$$

where $\phi=\frac{\pi}{2}-\Delta \phi_{x}-\Delta \phi_{y}$. From $\mathbf{F}$, we can derive the Lagrangian finite strain tensor $\mathbf{E}$ and local rotation matrix $\mathbf{R}$ by:

$$
\begin{aligned}
& \mathbf{E}=\frac{1}{2}\left(\mathbf{F}^{T} \cdot \mathbf{F}-\mathbf{I}\right) \\
& \mathbf{R}=\mathbf{F}\left(\mathbf{F}^{T} \cdot \mathbf{F}\right)^{-1 / 2}
\end{aligned}
$$

where $\mathbf{I}$ is an identity matrix.

Rather than the 2D horizontal-vertical Lagrangian strain tensor in Equation2, in myocardial deformation research, we are generally more interested in the radial and circumferential strains. Positive and negative radial strains indicate myocardial thickening and thinning, respectively, while myocardial stretching and shortening are represented by positive and negative circumferential strains, respectively. We define an angle $\theta$ about the centroid of the $\mathrm{LV}$, and transform $\mathbf{E}$ into a radial-circumferential strain tensor $\mathbf{E}$ with a rotation matrix $\mathbf{Q}(\theta)$, so that $\dot{\mathbf{E}}=\mathbf{Q E Q}^{T}$.

In Fig. 2. we show a visual comparison of our estimates of radial strain, circumferential strain and local rotation angle in a normal subject and a patient. 


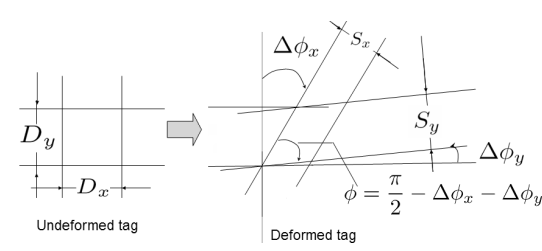

(a)

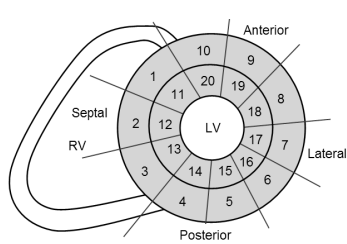

(b)

Fig. 1. (a): The illustration of $\mathbf{F}$ calculation. (b): The left ventricle is divided into 20 regions.

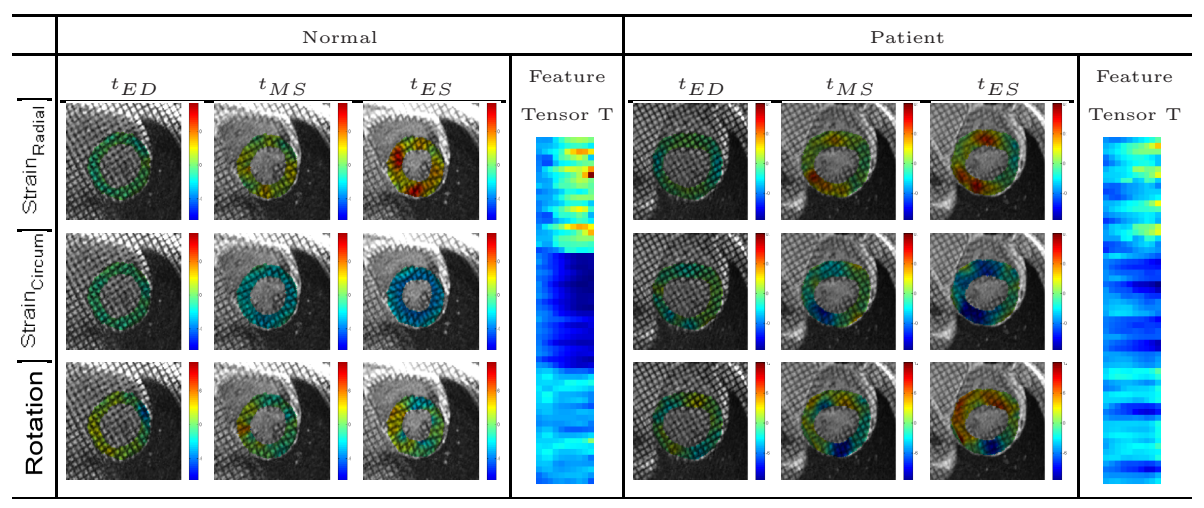

Fig. 2. Visual comparison of normal (left-hand-side) and patient's (right-hand-side) radial strain (first row), circumferential strain (second row) and rotation angle (third row) estimations at the time of ED, middle of systole (MS) and ES. For regional cardiac function analysis, we divide the left ventricle into 20 regions and interpolate the ED to $\mathrm{ES}$ deformation sequence into 10 frames, so as to form a $60 \times 10$-sized feature tensor $\mathbf{T}$, which is illustrated as a pseudo-color bar.

Warm color stands for higher strain value or bigger rotation angle. It is interesting to see that the magnitudes of the strain or rotation angle value are not the only criteria that can be used to diagnose cardiac abnormality. Their spatial and temporal distributions seem potentially more important. Normal hearts seem to have smoothly distributed strains and rotation angle values. This suggests that the myocardial function should be assessed on a comprehensive basis, including the strain magnitude, the regional strain pattern, the regional tissue rotation pattern, and their temporal evolution.

Quantitative data preparation. For quantitative regional cardiac function analysis, we divide the left ventricle at a given base-apex level into two layers (endo- and epi-cardium) and ten sectors (septum is equally divided into 3 sectors and the rest of the heart is divided into 7). This results in 20 ordered regions in total, as can been seen in Fig. 1 (b). Within each region, we use the median value of the radial strain, the circumferential strain and the rotation angle to 
represent this region's deformation, so as to remove outliers and make the data preparation more robust. We denote them as $\mathbf{E}_{R}^{i}, \mathbf{E}_{C}^{i}$ and $\theta^{i}(1 \leq i \leq 20)$, respectively. Therefore, the myocardial deformation in a single time frame can be represented by a $60 \times 1$-sized vector $\mathbf{V}=\left(\mathbf{E}_{R}^{1}, \ldots, \mathbf{E}_{R}^{20}, \mathbf{E}_{C}^{1}, \ldots \mathbf{E}_{C}^{20}, \theta^{1}, \ldots, \theta^{20}\right)^{T}$.

Due to the fading of the tags, myocardial deformation cannot be reliably extracted from frames after the end of systole (ES). Therefore, we only consider the image frames from end of diastole (ED) to ES. In addition, the heart beat rate varies in human subjects, and the absolute image frame rate varies between studies, so that the frame number from ED to ES ranges from 5 to 12 in our data. We implement a B-spline technique to interpolate them into 10 frames. Therefore, the myocardial motion of a tMRI time sequence can be represented by a $60 \times 10$-sized feature tensor $\mathbf{T}=\left[V_{1}, \ldots, V_{10}\right]$. As seen in Fig. 2 , the feature tensor $\mathbf{T}$ exhibits noticeable differences between normal subjects and patients.

\subsection{Spatio-temporal Tensor LDA}

Learn the classifier. Suppose we have collected $N$ samples (including normal and abnormal) for training purposes. We can train a typical two-class classifier to make the normal samples far away from the abnormal samples. LDA is a popular method to maximize the between-class scatter and minimize the within-class scatter, but it is a vector-based method. If we reshape the spatio-temporal tensor feature into a vector, it will collapse the spatio-temporal structure information, which is very important for identification. Additionally, the conventional LDA can only project the features into a scalar in the two-class case. In this paper, we propose to use spatio-temporal tensor LDA to deal with the above issues, as it can preserve the spatio-temporal information well, and it also has no limitation on dimensionality reduction.

Let $\mathbf{T}_{i}, i \in[1,2, \ldots, N]$ be training tensor features with the labels of normal and abnormal. Similar to LDA, the spatio-temporal tensor-based LDA (ST-LDA) also employs the Fisher criterion that maximizes the between-class scatter and minimizes the within-class scatter, but it extends the vector-based norm into the Frobenius norm [10]. With the tensor features $\mathbf{T}_{i} \in \mathfrak{R}^{60 \times 10}$, the betweenclass scatter $S_{b}$ and the within-class scatter $S_{w}$ measured by the Frobenius norm are $S_{b}=\sum_{i=1}^{2} N_{i}\left\|M_{i}-M\right\|_{F}^{2}$ and $S_{w}=\sum_{i=1}^{2} \sum_{\mathbf{T}_{j} \in X_{i}}\left\|\mathbf{T}_{j}-M_{i}\right\|_{F}^{2}$, where $N_{i}$ means the number of $i$-th class sample, $M$ is the mean matrix of all the $\mathbf{T}_{i}$, $M_{i}$ represents the mean matrix of the $i$-th class, and $\mathbf{T}_{j} \in X_{i}$ means that $\mathbf{T}_{j}$ belongs to the $i$-th class.

The goal is to find the optimal projection matrices $L \in \mathfrak{R}^{60 \times d_{L}}$ and $R \in$ $\mathfrak{R}^{10 \times d_{R}}$ which maximize $S_{b}$ and minimize $S_{w}$ in the low dimensional subspace of $L \otimes R$, i.e., maximizing $S_{b}^{\prime}=\sum_{i=1}^{2} N_{i}\left\|L^{T}\left(M_{i}-M\right) R\right\|_{F}^{2}$ and minimizing $S_{w}^{\prime}=\sum_{i=1}^{2} \sum_{\mathbf{T}_{j} \in X_{i}}\left\|L^{T}\left(\mathbf{T}_{j}-M_{i}\right) R\right\|_{F}^{2}$ at the same time.

Because $\|X\|_{F}^{2}=\operatorname{trace}\left(X X^{T}\right), S_{b}^{\prime}$ and $S_{w}^{\prime}$ can be written as $S_{b}^{\prime}=$ trace $\left(L^{T} D_{b}^{R} L\right)$ and $S_{w}^{\prime}=\operatorname{trace}\left(L^{T} D_{w}^{R} L\right)$ when $R$ is given, where

$$
D_{b}^{R}=\sum_{i=1}^{2} N_{i}\left(M_{i}-M\right) R R^{T}\left(M_{i}-M\right)^{T}
$$




$$
D_{w}^{R}=\sum_{i=1}^{2} \sum_{\mathbf{T}_{j} \in X_{i}}\left(\mathbf{T}_{j}-M_{i}\right) R R^{T}\left(\mathbf{T}_{j}-M_{i}\right)^{T}
$$

Then we can get the optimal projection $L$ by maximizing $\operatorname{trace}\left(\left(L^{T} D_{w}^{R} L\right)^{-1}\left(L^{T} D_{b}^{R} L\right)\right)$, i.e., computing the eigenvectors of $\left(D_{w}^{R}\right)^{-1} D_{b}^{R}$.

Similarly, if $L$ is fixed, we can rewrite $S_{b}^{\prime}$ and $S_{w}^{\prime}$ as $S_{b}^{\prime}=\operatorname{trace}\left(R^{T} D_{b}^{L} R\right)$ and $S_{w}^{\prime}=\operatorname{trace}\left(R^{T} D_{w}^{L} R\right)$, because $\operatorname{trace}(A B)=\operatorname{trace}(B A)$, where

$$
\begin{gathered}
D_{b}^{L}=\sum_{i=1}^{2} N_{i}\left(M_{i}-M\right)^{T} L L^{T}\left(M_{i}-M\right) \\
D_{w}^{L}=\sum_{i=1}^{2} \sum_{\mathbf{T}_{j} \in X_{i}}\left(\mathbf{T}_{j}-M_{i}\right)^{T} L L^{T}\left(\mathbf{T}_{j}-M_{i}\right)
\end{gathered}
$$

Then the optimal projection can be obtained by maximizing $\operatorname{trace}\left(\left(R^{T} D_{w}^{L} R\right)^{-1}\left(R^{T} D_{b}^{L} R\right)\right)$, i.e., solving for the eigenvectors of $\left(D_{w}^{L}\right)^{-1} D_{b}^{L}$.

Thus, the final optimal solution can be computed by an iterative procedure, as shown in Table 1. It can be found that the ST-LDA algorithm not only avoids the eigen-decomposition in the 600-dimensional space, but also well preserves the geometric relations of row and column of $\mathbf{T}_{i}$. In addition, in the conventional LDA, the available dimension has the upper bound $C-1$, which means it will project onto a scalar in our normal/abnormal two classes situation, while STLDA has no such constraint and is able to keep more information, which will be essential when we back-project the low dimensional subspace feature to the original feature space and look for the reasons for the classification.

For a new myocardial deformation pattern $\mathbf{T}_{\text {test }} \in \mathfrak{R}^{60 \times 10}$, its projection in the reduced dimensional subspace is: $Y_{\text {test }}=L^{T} \mathbf{T}_{\text {test }} R \in \Re^{d_{L} \times d_{R}}$. Classification is done in this subspace using a k-nearest neighbor scheme, where we empirically set $k=3$.

Table 1. The ST-LDA algorithm

\section{Input: $\mathbf{T}_{1}, \mathbf{T}_{2}, \ldots, \mathbf{T}_{N}$}

Initialization: Set $R_{0}=\left(I_{d_{R}}, 0\right)^{T}$, and compute the mean $M_{i}$ of the $i$-th class for each class, and the global mean $M$.

Iteration: For $t=1$ to $t_{\max }$

1). For a given $R_{t-1}$, compute $D_{w}^{R}$ and $D_{b}^{R}$ using Equations 5 and 4 , and get the optimal $L_{t}$ by solving for the first $d_{L}$ leading eigenvectors of $\left(D_{w}^{R}\right)^{-1} D_{b}^{R}$.

2). Based on $L_{t}$, compute $D_{w}^{L}$ and $D_{b}^{L}$ as in Equations 7 and 6 , and get the optimal $R_{t}$ by solving for the first $d_{R}$ leading eigenvectors of $\left(D_{w}^{L}\right)^{-1} D_{b}^{L}$.

3). If $t>1,\left\|L_{t}-L_{t-1}\right\|<\varepsilon$ and $\left\|R_{t}-R_{t-1}\right\|<\varepsilon$, break; else, continue.

\section{End iteration.}

Output: $L=L_{t_{\max }}$ and $R=R_{t_{\max }}$ 
Regional abnormality analysis. Identifying and localizing abnormal cardiac regions could be of more clinical value than just classifying a heart as normal or diseased. The intuition behind the ST-LDA algorithm is that the high dimensional feature tensor can be projected into a lower dimensional subspace that is optimal for classification. Therefore, the tensor distance between abnormal and normal features in the low dimensional subspace becomes a concise but accurate description of the abnormality. If we back-project this feature distance to the original space, we can recover the location of the pathology on the original spatio-temporal structure in a more physically meaningful way.

Because the linear projection functions $L$ and $R$ are not orthogonal matrices, the back-project operation needs to be done using their pseudo-inverse matrices $L^{+}$and $R^{+}$. Suppose $T_{a}$ is a feature tensor, and $Y_{a}$ is its projection in the low dimensional subspace, which is classified as abnormal using the ST-LDA classifier. Then the feature distance in the original space can be derived by:

$$
d T=\left(L^{+}\right)^{T}\left(Y_{a}-\bar{Y}_{\text {normal }}\right) R^{+}
$$

Since $d T \in \mathfrak{R}^{60 \times 10}$ and we are more interested in recovering the spatial distribution of the cardiac function, we define an index of pathology $P_{i}, i=1,2, \ldots, 20$ that indicates each cardiac region's degree of functional abnormality. Note that $P$ is a temporal and functional (including radial strain, circumferential strain and rotation angle) combination of the abnormal distances, therefore, it is no longer a descriptor of local strain or rotation angle, but rather a systematic indicator of the local cardiac function.

$$
P_{i}=\sum_{t=1}^{10}\left(d T^{2}(i, t)+d T^{2}(20+i, t)+d T^{2}(40+i, t)\right)^{\frac{1}{2}}
$$

\section{Experiments and Results}

We acquired 41 time sequences of short-axis tagged MR images from 10 normal subjects and 12 patients. The patient's heart diseases varied from infarction or hypertrophy to general loss of myocardial function. The spatial positions of these SA images are confined to the mid-portion of the left ventricle, where the pathologies are usually prominent. In the ST-LDA classification algorithm, we empirically set the iteration number to 10 , and the dimension of the subspace to $10 \times 5$, so that $L \in \mathfrak{R}^{60 \times 10}$ and $R \in \mathfrak{R}^{10 \times 5}$. To make comparisons with other conventional classification methods, we applied PCA, LDA and SVM algorithms to the same data set. Their parameters are also empirically set to be optimal.

The training and testing procedures are strictly done on a leave-one patientout-basis, i.e., we leave one patient data for testing, and use the rest for training. In Table 2, we list the accuracy rate comparison of PCA, LDA, SVM and our proposed ST-LDA. We find that our novel algorithm outperforms PCA and LDA in accuracy, and has a similar accuracy rate with the nonlinear approach SVM. On the other hand, PCA and ST-LDA is able to recover the regional abnormality 
Table 2. Comparison with other conventional classification algorithms

\begin{tabular}{r||c|c|c||c}
\hline \hline Classification Algorithm & PCA & LDA & SVM & ST-LDA \\
\hline Classification Accuracy Rate & $73.17 \%$ & $85.37 \%$ & $87.80 \%$ & $87.80 \%$ \\
\hline \hline
\end{tabular}
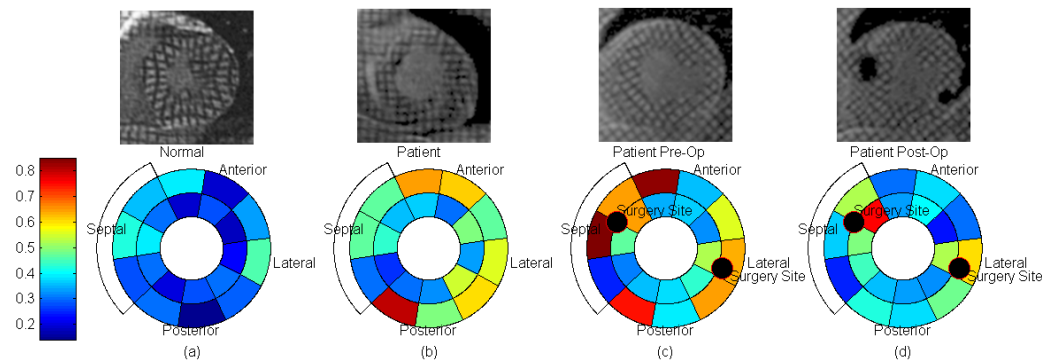

Fig. 3. Quantitative analysis of the regional abnormality from the back-projection. Warm color represents high $P$ value, which means high degree of abnormality. The first row shows the sampling images at ES from 4 time sequences. The second row shows the analysis results. Column (a) is from a normal sequence. Column (b) is from an abnormal subject. (c) and (d) are from a patient before and after a surgery, respectively. The black dots show the surgery sites.

by back-projecting the subspace feature to the original feature space, while LDA and SVM cannot.

In Fig. 3. we show some representative results of the regional cardiac function analysis. The regional abnormality is represented by the index $P$, which is encoded in the pseudo color map. Column (a) is from a normal sequence. We can observe that the $P$ index is low and smoothly distributed. Column (b) is from an abnormal subject. The warm color indicates possible abnormal regions, such as the 1 and 5 o'clock regions, which agree with doctor's diagnosis. The high abnormality at the 7 o'clock region very likely comes from the LVRV connecting area, whose motion is affected by both ventricles and therefore sensitive to pathology. Columns (c) and (d) are from a patient before and after a surgery, respectively. This patient had insertion of a "Coapsys" device to reduce functional mitral regurgitation. We can observe that the doctor's diagnosis (indicated by the surgical sites, which appear as dark dots in the MR image, and depicted as black dots in the analysis results) coincides with the abnormal regions from our analysis results very well. Furthermore, the overall performance is more normal as depicted in the overall cooler color in $(\mathrm{d})$, which shows the effectiveness of this surgery. However, the myocardium at the two surgical sites were impaired in the surgery, which is why the cardiac function is still (or even more) abnormal near the surgical sites in (d). Our regional cardiac function analysis results perfectly agree with the patient's pathologies before and after the operation. 


\section{Conclusion}

In this paper, we have proposed a novel spatio-temporal tensor-based LDA classification framework to detect and localize regional cardiac abnormality. The advantage of our proposed method is that its dimension reduction is separately operated in the spatial and temporal domains, so that it conserves better the spatio-temporal structure and information of the training data. In addition, in order to analyze regional cardiac function, we back-project the abnormality distance found in the subspace to the original feature space, so as to localize the regional abnormality in a more physically meaningful way. Our experimental results show our ST-LDA approach achieves a higher classification rate than conventional linear approaches, and achieves comparable accuracy rate to nonlinear methods, such as SVM. The recovered regional abnormalities agree with the doctor's diagnosis and patient's pathology very well.

\section{References}

1. Axel, L., Dougherty, L.: MR imaging of motion with spatial modulation of magnetization. Radiology 171, 841-845 (1989)

2. Axel, L., Chen, T., Manglik, T.: Dense myocardium deformation estimation for 2D tagged MRI. In: Frangi, A.F., Radeva, P.I., Santos, A., Hernandez, M. (eds.) FIMH 2005. LNCS, vol. 3504, pp. 446-456. Springer, Heidelberg (2005)

3. Clarysse, P., Basset, C., Khouas, L., Croisille, P., Friboulet, D., Odet, C., Magnin, I.E.: 2D spatial and temporal displacement and deformation field fitting from cardiac MR tagging. Medical Image Analysis 4, 253-268 (2000)

4. Osman, N.F., McVeigh, E.R., Prince, J.L.: Imaging heart motion using harmonic phase MRI. IEEE Trans. Med. Imaging 19(3), 186-202 (2000)

5. Park, J., Metaxas, D., Young, A., Axel, L.: Deformable models with parameter functions for cardiac motion analysis. IEEE Transactions on Medical Imaging 15(3), 278-289 (1996)

6. Hu, Z., Metaxas, D.N., Axel, L.: In-vivo strain and stress estimation of the left ventricle from MRI images. In: Dohi, T., Kikinis, R. (eds.) MICCAI 2002. LNCS, vol. 2488, pp. 706-713. Springer, Heidelberg (2002)

7. Declerck, J., Feldmar, J., Ayache, N.: Definition of a 4d continuous planispheric transformation for the tracking and the analysis of LV motion. Medical Image Analysis 2(2), 197-213 (1998)

8. Clarysse, P., Han, M., Croisille, P., Magnin, I.E.: Exploratory analysis of the spatiotemporal deformation of the myocardium during systole from tagged MRI. IEEE Trans. Biomed. Eng. 49, 1328-1339 (2002)

9. Qian, Z., Metaxas, D., Axel, L.: Non-tracking-based 2d strain estimation in tagged MRI. In: Proc. of the IEEE International Symposium on Biomedical Imaging (2007)

10. Ye, J.P., Janardan, R., Li, Q.: Two-dimensional linear discriminant analysis. In: Proc. of. Int. Conf. Neural Information Processing Systems (2004) 